\title{
Patientenverfügungen - eine Herausforderung für Patient und Arzt
}

\author{
C. Marti, U. Bovet
}

Patientenverfügungen helfen Angehörigen, Pflegepersonen und uns Ärztinnen und Ärzten, Menschen auch dann noch in ihrem Sinn zu betreuen und zu behandeln, wenn sie selber nicht mehr entscheidungsfähig sind. Das Erarbeiten einer Patientenverfügung und der Umgang damit bedeuten eine Herausforderung für alle Beteiligten und Betroffenen. Liegt keine Verfügung vor, kann es zu belastenden Konflikten zwischen Angehörigen, Pflegepersonen und betreuenden Ärzten kommen.

\footnotetext{
* Die Ärztegruppe WintiMed betreut seit 1994 einen Teil ihrer Patienten im Rahmen von alternativen Versicherungsmodellen, welche eine gemeinsame Budgetverantwortung beinhalten. Darum trifft sie sich regelmässig im Rahmen von moderierten und protokollierten Teamsitzungen zur Behandlung von medizinischen, organisatorischen und finanziellen Fragen und Projekten. Der Konsens betreffend Patientenverfügung wurde an einem halbtägigen Workshop sowie an mehreren Teamsitzungen erarbeitet und am 19. November 2003 mit etwa 100 interessierten Laien in moderierten Gruppen diskutiert. Wir danken Christoph Wahl, leitender Arzt am KS Winterthur, für seine Mitarbeit und Jakob Elmer, Unternehmensberater, der uns bei der Konsensfindung behilflich war.
}

Korrespondenz:

Dr. med. Christian Marti

WintiMed

Rosinliweg 44

CH-8400 Winterthur
Wer vor dreissig Jahren in den Arztberuf einstieg, der begegnete keinen Patientenverfügungen. Auch heute sind sie noch eine Ausnahme in Praxis und Spital. Trotzdem gewinnen sie aus verschiedenen Gründen zunehmend an Bedeutung.

«Mein Wille geschehe», so überschrieb kürzlich die Zeitschrift «Stern» eine Recherche zum Thema Patientenverfügungen [1]. Dieser Titel weist auf einen Kulturwandel hin, nämlich die wachsende Anerkennung der Patientenautonomie neben dem traditionellen und immer noch dominierenden Paternalismus der ärztlichen Ethik. Ein weiterer Grund für die wachsende Bedeutung von Patientenverfügungen ist der rasante medizinische Fortschritt, welcher der Ärzteschaft eine Vielzahl von Behandlungsmöglichkeiten bietet. Weil oft unklar ist, ob diese eher eine Verlängerung des Lebens oder eine Verlängerung des Sterbens bewirken, stehen wir Ärztinnen und Ärzte zunehmend vor belastenden Ermessensentscheiden. Immer öfter überleben Menschen zwar einen schweren Unfall, bleiben aber dauernd bewusstlos. Vor allem aber gibt es immer mehr Menschen, die geistig so stark abgebaut haben, dass sie nicht mehr selber mitreden und entscheiden können, wenn es um die eigene medizinische Behandlung geht.

In einer Patientenverfügung halten Menschen schriftlich fest, welche Anliegen sie an die Angehörigen sowie an die betreuenden Ärzte und Pflegepersonen haben für den Fall, dass sie nicht mehr selber in der Lage sind, diese mitzuteilen (z.B. wegen Bewusstlosigkeit, geistigen Abbaus usw.). Unter anderem betreffen diese Anliegen oft das Ausmass und die Grenzen von medizinischen Bemühungen bei schweren, unheilbaren Krankheiten ohne Aussichten auf Besserung.
Die Ärztegruppe WintiMed hat sich im vergangenen Jahr intensiv mit Patientenverfügungen befasst*. Wir sind zum Schluss gekommen, dass Patientenverfügungen für uns betreuende Ärztinnen und Ärzte eine wertvolle Entscheidungshilfe und wirksame Entlastung sein können, denn sie helfen uns, aber auch den Angehörigen und allen beteiligten Betreuungspersonen, Menschen auch dann noch in ihrem Sinn $\mathrm{zu}$ betreuen und $\mathrm{zu}$ behandeln, wenn sie selber nicht mehr entscheidungsfähig sind, z.B. bei der Frage, ob eine Spitaleinweisung zur Therapieintensivierung im Sinne des Patienten ist.

Ausserdem haben wir uns auf folgenden Konsens geeinigt:

1. Wir WintiMed-Ärztinnen und -Ärzte respektieren die Patientenverfügungen unserer Patienten/-innen. Wir setzen uns dafür ein, dass sie auch von den Spezialärztinnen und -ärzten, von den Heim- und Spitalärztinnen und -ärzten sowie von den Angehörigen beachtet werden.

2. Stehen eine Patientenverfügung und die damit verknüpften Erwartungen an eine bestimmte WintiMed-Ärztin im Widerspruch zur persönlichen Haltung dieser WintiMedÄrztin (z.B. Freitodbegleitung), so weist sie ihre Patientin frühzeitig darauf hin und versucht, gemeinsam mit ihr einen für beide gangbaren Weg zu finden.

3. Wir WintiMed-Ärztinnen und -Ärzte sind gerne bereit, unsere Patienten/-innen zu beraten, wenn sie eine Patientenverfügung verfassen wollen.

4. Schriftliche Vorlagen und Anregungen für eine persönliche Patientenverfügung stellen jede WintiMed-Praxis sowie die WintiMedGeschäftsstelle zur Verfügung [2].

Das Erarbeiten einer Patientenverfügung ist eine grosse persönliche Herausforderung, denn sie verlangt die Auseinandersetzung mit dem eigenen Sterben und dem Tod. Wir ermuntern unsere Patienten, ihre Verfügung mit den nächsten Angehörigen und dem Arzt ihres Vertrauens zu besprechen und diesen eine Kopie zu übergeben. Denn eine Patientenverfügung, von der niemand weiss, nützt nichts. 
Fehlt eine Verfügung, so kann es zu belastenden Konflikten zwischen Angehörigen, Pflegepersonen und Ärzten kommen. Erst kürzlich berichteten Medien in aller Welt über eine Frau in Florida, die nach einem Herzstillstand seit Jahren bewusstlos am Leben erhalten wird. Ihrem Ehemann soll sie in gesunden Tagen gesagt haben, dass sie keine lebensverlängernden Massnahmen wünscht, sollte sie sich einmal in einem solchen Zustand befinden. Der Ehemann und die behandelnden Ärzte möchten deshalb die Behandlung abbrechen und die Patientin sterben lassen. Die Eltern der Patientin sind damit nicht einverstanden. Nun streiten Ehemann und Eltern der Patientin vor Gericht miteinander über Weiterführung oder Beendigung der Behandlung [1].

Selbst wenn eine Patientenverfügung vorliegt, sind schwerwiegende Konflikte zwischen Angehörigen und Ärzten möglich. So suchte eine Frau mit ihrem Vater, der zwei Jahre zuvor nach dem qualvollen Krebstod seiner Schwester eine Patientenverfügung verfasst hatte, meine Sprechstunde auf. Unter anderem hielt er fest, dass man «der Krankheit ihren natürlichen Lauf lassen» solle, falls er einmal geistig stark abgebaut sei, «z. B. wegen Alzheimer-Krankheit». Ein Jahr später erkrankte er an einem bösartigen Hirntumor, der einen raschen Zerfall der Persönlichkeit und die Einweisung in ein Pflegeheim zur Folge hatte. Die behandelnden Ärzte leiteten eine Chemotherapie ein, obwohl die Tochter sich unter Berufung auf die Patientenverfügung dagegen wehrte. Die Chemotherapie stoppte das Wachstum des Hirntumors, aber dem Patienten ging es kein bisschen besser. Als die Tochter sich gegen die Fortsetzung der Chemotherapie wehrte, wurden ihr persönliche Interessen am raschen Tod des Vaters unterstellt. Diese Geschichte zeigt, dass der Umgang mit Patientenverfügungen Ärzte überfordern kann [3].

Mit diesem Beitrag möchten wir darum die Diskussion in Ärzteschaft und Öffentlichkeit über Nutzen und Verbindlichkeit von Patientenverfügungen anregen.

\section{Literatur}

1 Schmitz T. Mein Wille geschehe? Stern 2003; 47:196-203.

2 Humandokument des interdisziplinären Instituts für Ethik im Gesundheitswesen «Dialog Ethik» (www.dialog-ethik.ch) sowie Vorlage der Redaktion Puls-Tipp (www.konsuminfo.ch/downloads. asp).

3 Marti C, Oelz O, Muffler M, Bärtschi K. Missachtete Patientenverfügung und Case-Management. Managed Care 2003;5:28-32. 